\title{
High-throughput Separation of White Blood Cells from Whole Blood using Inertial Microfluidics
}

\author{
Jun Zhang, Member, IEEE, Dan Yuan, Ronald Sluyter, Sheng Yan, Qianbin Zhao, Huanming Xia, Say \\ Hwa Tan, Nam-Trung Nguyen, Member, IEEE, Weihua Li, Member, IEEE
}

\begin{abstract}
White blood cells (WBCs) constitute only about $0.1 \%$ of human blood cells, yet contain rich information about the immune status of the body, thus separation of WBCs from the whole blood is an indispensable and critical sample preparation step in many scientific, clinical and diagnostic applications. In this work, we developed a continuous and high-throughput microfluidic WBC separation platform utilizing the differential inertial focusing of particles in serpentine microchannels. First, separation performance of the proposed method is characterized and evaluated using polystyrene beads in the serpentine channel. The purity of $10-\mu \mathrm{m}$ polystyrene beads is increased from $0.1 \%$ to $80.3 \%$ after two cascaded processes, with an average enrichment ratio of 28 times. Next, we investigated focusing and separation properties of Jurkat cells spiked in the blood to mimic the presence of WBCs in whole blood. Finally, separation of WBCs from human whole blood was conducted and separation purity of WBCs was measured by the flow cytometry. The results show that the purity of WBCs can be increased to $48 \%$ after two consecutive processes, with an average enrichment ratio of 10 times. Meanwhile, a parallelized inertial microfluidic device was designed to provide a high processing flow rate of $288 \mathrm{ml} / \mathrm{h}$ for the diluted $(\times 1 / 20)$ whole blood. The proposed microfluidic device can potentially work as an upstream component for blood sample preparation and analysis in the integrated microfluidic systems.

Index Terms-Inertial microfluidics, particle focusing and separation, blood cell separation, white blood cells, leukocytes, serpentine microchannnel
\end{abstract}

This work is supported in part by the Natural Science Foundation of Jiangsu Province (BK20170839) and the University of Wollongong-China Scholarship Council joint scholarships.

J. Zhang and H. Xia are with School of Mechanical Engineering, Nanjing University of Science and Technology, Nanjing, Jiangsu 210094, China (e-mail: junzhang@njust.edu.cn; hmxia@njust.edu.cn)

D. Yuan, S. Yang, Q. Zhao and W.H. Li are with School of Mechanical, Materials and Mechatronic Engineering, University of Wollongong, Wollongong, NSW 2522, Australia ( e-mail: dy983@uowmail.edu.au; sy034@uowmail.edu.au ; qz260@uowmail.edu.au ; weihuali@uow.edu.au

R. Sluyter is with School of Biological Sciences and Illawarra Health and Medical Research Institute, University of Wollongong, Wollongong, NSW 2522, Australia(e-mail: rsluyter@uow.edu.au)

N-T Nguyen and S-H Tan are with Queensland Micro and Nanotechnology Centre, Griffith University, Brisbane QLD 4111, Australia (e-mail: nam-trung.nguyen@griffith.edu.au; sayhwa.tan@griffith.edu.au)

\section{INTRODUCTION}

LOOD contains massive information about the functioning and status of the body. Separation of blood sample is a requisite and critical step in many research applications, and clinical diagnoses and therapeutics [1-5]. Current methods for blood cell separation require complex and expensive equipment. Centrifugation, the "gold standard" and the most common method for blood cell separation, is labor-, energy- and time-intensive and relies on well-trained operators $[6,7]$. Another common approach is to expose whole blood to the hypotonic solution to lyse red blood cells (RBCs), which are more sensitive to osmotic shock than nucleated white blood cells (WBCs) [8]. In this method, centrifugation is still required to remove the lysed RBC debris from WBCs. Moreover, exposure to a high acceleration or osmotic shock will probably influence the immunophenotype [9] and viability[10, 11] of the cells.

Microfluidics has been emerging as a powerful technology and holds the potential for high resolution and high efficient separation of blood cells in microchannels. In microfluidic devices, active external (electric, acoustic and magnetic) force fields were applied to facilitate the fractionation of blood cells. Han and Frazier [12] presented lateral-driven continuous dielectrophoretic (DEP) microseparators to separate RBCs and WBCs from diluted $(\times 1 / 6)$ whole blood at a flow rate of 50 $\mu \mathrm{l} / \mathrm{min}$. The separation efficiency of WBCs for the divergent and convergent type DEP microseparators was $92.1 \%$ and $76.9 \%$, respectively. Meanwhile, free flow acoustophoresis (FFA) was proposed and developed to separate polystyrene particles and blood cells [13]. This method has been further developed to fractionate WBCs subpopulations (lymphocytes, monocytes, and granulocytes) [14]. For lymphocytes and granulocytes, flow cytometry data reveal high purity $(95.2 \%$ and $98.5 \%$, respectively) and high recovery (86.5\% and $68.4 \%$, respectively). However, despite a high recovery $(83.1 \%)$, a relatively low purity $(25.2 \%)$ was obtained for monocytes. In addition, employing intrinsic differences in magnetic property between WBCs and RBCs can also enable the separation of WBCs and RBCs. Han and Frazier [15] developed continuous cascade paramagnetic capture (PMC) mode microfluidic separators for high efficient separation of RBCs and WBCs based on the native magnetic properties. The PMC microseparator continuously separated $93.5 \%$ of RBCs and $97.4 \%$ of WBCs from whole blood at a volumetric flow 
rate of $5 \mu \mathrm{l} / \mathrm{h}$. Moreover, Iliescu et al. [16] reported a microfluidic device for magnetophoretic capture of RBCs from blood under continuous-flow condition. The ferromagnetic wires generate a gradient of magnetic field which is perpendicular to the flowing direction and enlarges the magnetic force to capture RBCs on the bottom of the microfluidic channel, while the rest of the blood flows through and is collected at the outlet. Experimental results show that 95\% of RBCs were trapped in the device.

Meanwhile, fractionation of blood cells by employing intrinsic hydrodynamic force or cell-cell interactions without external force fields was also reported. Cross flow filtration which filtrates small particles from large ones by an array of side branches has been developed for blood cell separation [17-20]. Yamada and Seki [17] proposed a hydrodynamic filtration in a microchannel with multiple side branch channels to concentrate WBCs from $(\times 1 / 10)$ diluted blood samples at a flow rate of $20 \mu \mathrm{l} / \mathrm{min}$. The ratio of WBCs to RBCs was increased from $0.13 \%$ to $3.71 \%$ after two rounds of filtration. In addition to side branch, the surface membrane was integrated within a crossflow filtration scheme in a microfluidic chip to sort WBCs from whole blood [19]. The recovery rate of WBCs was $27.4 \%$ and purity was $93.5 \%$ with a sample-throughput of $1 \mathrm{ml} / \mathrm{h}$.

WBC margination, which is recognized as an outcome of the cell deformability and shape differences between RBCs and WBCs, is also used for blood separation [8, 21]. Jain et al. [8] developed a biomimetic postcapillary expansion technique using repeated triangular expansions/contractions to extract nucleated cells at a rate of $18 \mu \mathrm{l} / \mathrm{h}$ on the unprocessed whole blood. The recovery rate of $94 \%$ and purity of $5 \%$ were obtained. By patterning slanted ridges on the top of the straight channel, Kim et al. [21] developed a microfluidic platform to achieve deterministic separation of WBCs from whole blood, achieving high-throughput separation of cells $(150 \mu \mathrm{l} / \mathrm{min}$ for undiluted whole blood). The ratio of WBCs to RBCs was 110:1000 in the WBCs reservoir after multistage $(n=4)$ device operation, with a recovery rate of $80 \%$.

Recently, inertial microfluidic technology which manipulates microparticles by the finite inertial effects of fluid and particles has attracted significant attention due to the advantages of simple structure, high throughput, and precise manipulation [22-26]. Wu et al. [27] presented a novel inertial platform for continuous sheathless particle and blood cell separation in straight microchannels containing expansion-contraction microstructures. The processing rate for the device with 72 parallel channels for diluted $(\times 1 / 400)$ blood was $10.8 \mathrm{ml} / \mathrm{min}$, with separation purity and efficiency as high as $\sim 90 \%$. Spiral microchannels, which induce Dean flow to modify inertial focusing and facilitate cell separation, were proposed and applied for blood cell separation. Nivedita and Papautsky [28] demonstrated the continuous separation of RBCs and WBCs from a diluted $(\times 1 / 500)$ sample of blood in a spiral microchannel at a flow rate of $1.8 \mathrm{ml} / \mathrm{min}$, equivalent to the flow rate of $3.6 \mu 1 / \mathrm{min}$ for undiluted whole blood. Moreover, $\mathrm{Wu}$ et al. [29] presented a novel design of a spiral inertial microfluidic device with a trapezoidal cross-section for high efficient WBCs separation. For $0.5 \%$ hematocrit blood sample, $\sim 98.4 \%$ of total WBCs $(99.4 \%$ granulocytes and $92.4 \%$ mononuclear leukocytes) were recovered at a throughput of $\sim 900 \mu \mathrm{l} / \mathrm{min}$, equivalent to $10 \mu \mathrm{l} / \mathrm{min}$ for whole blood (at $45 \%$ hematocrit). However, the throughput of these inertial microfluidic devices is still on the order of $\sim 10 \mu \mathrm{l} / \mathrm{min}$ for the undiluted blood, which is not desirable for large volume sample preparation.

In the present work, we developed a continuous and high-throughput inertial microfluidic device with symmetric serpentine microchannels to further improve the throughput. The separation principle is based on the differential inertial focusing of particles in a microchannel. First, we characterized and evaluated the separation performance of the proposed methods using polystyrene beads. The purity of $10-\mu \mathrm{m}$ polystyrene beads was increased from $0.1 \%$ to $80.3 \%$ after two processes, with an average purity enrichment ratio of 28 times. Next, Jurkat cells, a human T lymphocyte cell line, were spiked in the blood sample to mimic the presence of WBCs in whole blood, and its focusing and separation performance in the whole blood was investigated in the proposed microchannel. Finally, separation of WBCs from diluted whole blood was conducted in a cascaded manner, and flow cytometry was used to characterize the separation purity of WBCs. A parallelized inertial microfluidic device was designed and fabricated to provide a high processing flow rate of $288 \mathrm{ml} / \mathrm{h}$ for the diluted $(\times 1 / 20)$ whole blood. We envision that the parallelized serpentine channels could be a useful alternative to conventional centrifugation for high efficient and high-throughput RBC depletion and WBC purification.

\section{METHODS AND MATERIALS}

\section{A. Device Design and Fabrication.}

The microchannel in the experiments is $15.2 \mathrm{~mm}$ long, comprised of 15 zigzag periods. The depth and width of the microchannel are $42 \mu \mathrm{m}$ and $200 \mu \mathrm{m}$, respectively. The width and length of each U-turn are both $700 \mu \mathrm{m}$. At the end of the serpentine channel, a trifurcation outlet is implemented, and the two-sided symmetrical branches are merged for an easy fluid collection. In order to amplify the processing throughput, eight serpentine channels were parallelized along the planar direction in a single microfluidic device. All components were fabricated by the standard photolithography and PDMS soft lithography techniques [30].

\section{B. Particle and Cell Preparation}

Polystyrene particles internally stained with fluorescent dyes were obtained from Thermo Fisher Scientific. Particles with diameters of $3 \mu \mathrm{m}$ (Product No. R0300, CV5\%) and 10 $\mu \mathrm{m}$ (Product No. G1000, CV5\%) were respectively dispersed in deionized (DI) water and the particle weight ratio is about $0.05 \%$. Tween 20 (Sigma-Aldrich, Product No. P9416) with was added in the DI water as a surfactant at a weight ratio of $0.1 \%$ to avoid particle aggregation. For the separation test, a mixture of polystyrene beads was prepared by mixing $3-\mu \mathrm{m}$ and $10-\mu \mathrm{m}$ particles to a final concentration of $4 \times 10^{7}$ counts $/ \mathrm{ml}$ and $4 \times 10^{4}$ counts/ml in the DI water, respectively.

For cell experiments, Jurkat cells, an immortalized line of human $\mathrm{T}$ lymphocytes, were cultured in Roswell Park Memorial Institute (RPMI) 1640 medium (ThermoFisher Scientific) containing $10 \%$ fetal bovine serum (Bovogen Biologicals) and 1\% L-glutamine (ThermoFisher Scientific) 
within a humidified $5 \% \quad \mathrm{CO}_{2}, 37{ }^{\circ} \mathrm{C}$ incubator (Thermo Scientific) using the standard protocols. Jurkat cells, which grow in the culture medium, were centrifuged at $450 \times \mathrm{g}$ for 5 min. After aspirating the supernatant, the cells pellet was resuspended gently in a pre-warmed CellTracker Green CMFDA solution (Product NO. C2925, CellTracker) and incubated for $30 \mathrm{~min}$. Finally, the stained cells were centrifuged at $450 \times \mathrm{g}$ for $5 \mathrm{~min}$, diluted in the phosphate-buffered saline (PBS) to the desired concentration of $\sim 1 \times 10^{5}$ cells $/ \mathrm{ml}$, or spiked in whole blood to mimic the presence of WBCs. The cells were stained to facilitate observation of cell trajectory in the serpentine microchannel and counting of recovered cells. Whole blood was collected from human volunteers into lithium heparin tubes (Greiner Bio-One). Blood sample was diluted 20 times with PBS before being infused into the microfluidic device.

\section{Experimental Setup and Method}

The microfluidic devices were located on the stage of an inverted fluorescence microscope (CKX4, Olympus). A syringe pump (Legato 100, KD Scientific) was used to control the flow rate and infuse particle suspension and blood sample into the microfluidic devices. An high-speed CCD camera (Optimos, Q-imaging) mounted on the microscope recorded the trajectories of particles and cells, and the captured images were then post-processed and analyzed by a commercial image analysis software (Q-Capture Pro 7, Q-imaging). The experimental setup is shown in Fig. 1(a). The concentrations of polystyrene beads and blood cells were measured using a hemocytometer. The purity of polystyrene beads mixture collected from outlets after two cascaded processes was calculated based on three measurements. An LSR II flow cytometer (BD Biosciences) measured the Forward scatter (FSC) and side scatter (SSC) of blood samples before and after the process. FlowJo software (Tree Star) was used to analyze the data and evaluate the ratio of blood cell subpopulations.

\section{THEORY AND MECHANISM}

\section{A. Inertial Migration}

Particle inertial migration in straight channels is induced by the two inertial effects: the shear gradient lift force $F_{L S}$ and the wall lift force $F_{L W}$. The shear gradient lift force $F_{L S}$ originates from the parabolic fluid velocity profile and finite particle size, directing toward the channel wall. And the wall lift force $F_{L W}$ arises when particles are near channel wall, always pointing to the channel centerline. The particle focusing positions are the locations where both inertial lift forces counteract each other. If the particle size is far smaller than the channel size, the net inertial lift force $F_{L}$ can be expressed as following [22, 31]:

$$
\begin{gathered}
F_{L}=\frac{\rho_{f} U_{m}^{2} a^{4}}{D_{h}^{2}} f_{L}(\operatorname{Re}, z) \\
\operatorname{Re}=\frac{\rho_{f} U_{m} D_{h}}{\mu}
\end{gathered}
$$

where $a$ is particle diameter, and $D_{h}$ is the hydraulic diameter of the channel. $\rho_{f}, \mu$ and $U_{m}$ are the fluid density, dynamic viscosity, and maximum velocity, respectively. $f_{L}(R e, z)$ is the dimensionless coefficient of the inertial lift force, which is a function of the particle cross-sectional position $z$ and channel Reynolds number $\operatorname{Re}[22]$.

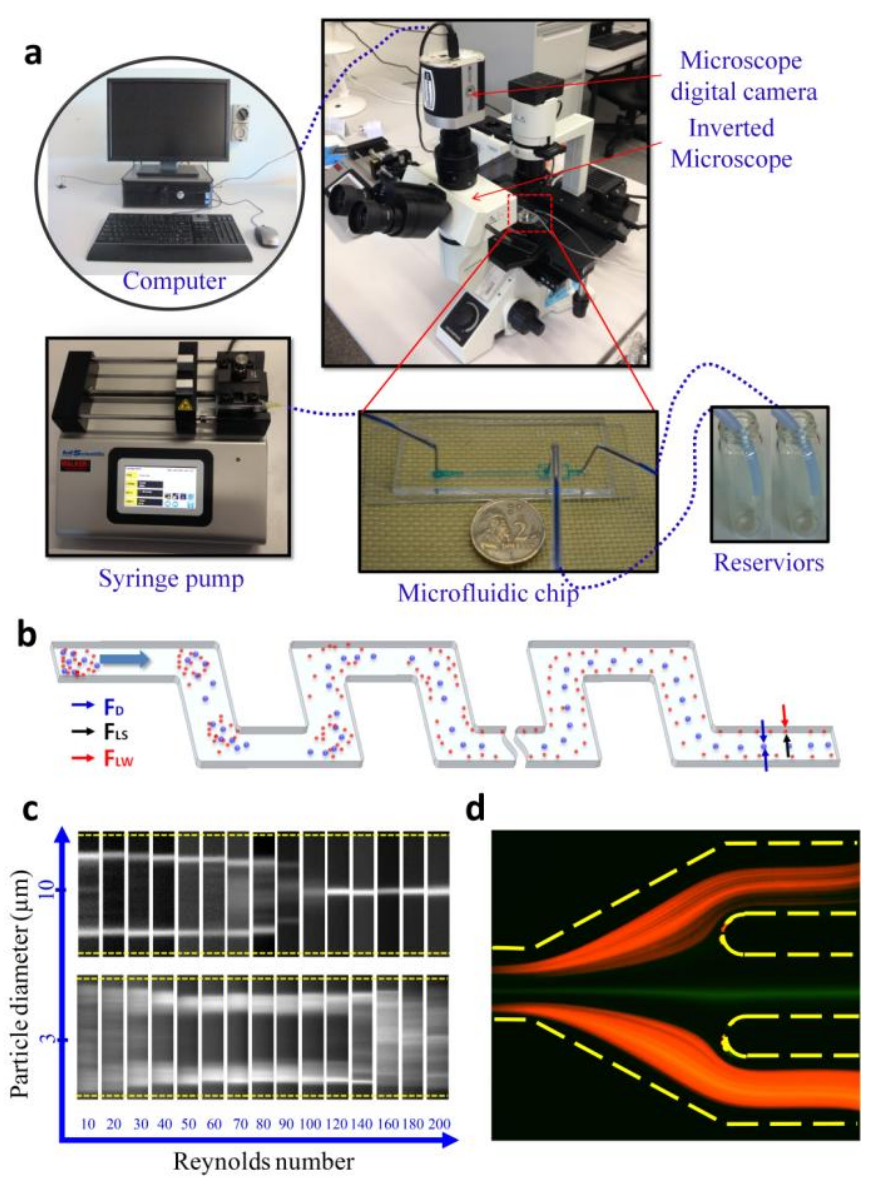

Fig. 1. (a) A diagram of experimental setup. The microfluidic chip is placed on an inverted microscope which is mounted with a digital camera. A syringe pump is used to control and infuse particle/cell suspension into the microfluidic chip at a specific flow rate, and the trajectories of particles/cells are recorded by the digital CCD camera and analyzed by the Q-capture Prof 7 software. (b) Inertial focusing and separation of microparticles in the symmetric serpentine microchannel. (c) The inertial equilibrium position of $3-\mu \mathrm{m}$ and $10-\mu \mathrm{m}$ polystyrene beads under different flow Reynolds numbers in the serpentine microchannel. (d) Separation of 3- $\mu \mathrm{m}$ (red) and 10- $\mu \mathrm{m}$ (green) polystyrene beads by differential equilibrium positions.

\section{B. Secondary Flow}

In a curving channel, fluid momentum mismatch in the center and near-wall region within the curvature causes a pressure gradient along the radial direction, which subsequently induces a secondary flow [22]. The fluid elements near the channel centerline flow outwards due to a higher momentum than those near the wall, and push the relatively stagnant fluid elements near the wall inwards along the circumference, thus producing two counter-rotating streams, also called Dean vortex [22]. The strength of the secondary flow $U_{D}$ is approximated as $[32,33]$ :

$$
U_{D}=1.8 \times 10^{-4} D e^{1.63}
$$

where the Dean number $D e$ is a function of the Reynolds number $R e$, the radius of the channel curvature $R$, and the hydraulic diameter of the channel $D_{h}$ [34]:

$$
D e=\sqrt{\frac{D_{h}}{2 R}} \operatorname{Re}
$$


For a particle moving at a speed of $U_{p}$, the Dean drag force $F_{D}$ on the particle can be calculated using Stokes' drag law [35]:

$$
F_{D}=3 \pi \mu a\left(U_{D}-U_{P}\right)
$$

The additional lateral forces from the secondary flow induced by the channel curvature could assist and modify inertial focusing process and positions [36]. The particle focusing pattern in the microchannel (i.e. the position, number, and bandwidth of the particle positions) can be analyzed by comparing the relative strength of the secondary flow drag and the inertial lift force [22]. If the secondary flow in the serpentine channel is too weak to offset the inertial lift force, the focusing pattern is still dominated by the inertial lift force, and particles are normally ordered along the two sidewalls [37]. If the secondary flow is strong enough, it will modify the particle focusing positions. In such case, a single stream can be obtained in a symmetric serpentine channel [38]. Benefitting from the property that the relative strength of these forces is highly dependent on particle size, particle separation by size in the serpentine channel can be obtained by the overlap of the two different regions.

\section{RESULTS AND DISCUSSION}

\section{A. Inertial Focusing of Polystyrene Beads}

Blood cells consist of three main classes: (i) leukocytes or WBCs, spherical cells $8-12 \mu \mathrm{m}$ in diameter, and concentration is $\sim(5-10) \times 10^{6}$ counts $/ \mathrm{ml}$; (ii) erythrocytes or RBCs, discoid, anuclear cells $\sim 8 \mu \mathrm{m}$ in diameter and $\sim 2.5 \mu \mathrm{m}$ in thickness, and concentration is $\sim 5 \times 10^{9}$ counts $/ \mathrm{ml}$; and (iii) platelets, discoid particles $1-3 \mu \mathrm{m}$ in diameter, and concentration is $(2-5) \times 10^{8}$ counts/ml $[18,39,40]$. The significant size difference of WBCs with other blood cells makes the size-based separation of WBCs possible.

As a first step, the rigid spherical polystyrene beads with similar size to relevant blood cells were used to characterize the inertial focusing behavior (e.g., inertial equilibrium positions and distribution) in the symmetrical serpentine microchannel. Initially, particles are focused as two streaks along the sidewalls. Increasing Reynolds numbers from 10 to 200 (corresponding to flow rate of $50-1000 \mu \mathrm{l} / \mathrm{min}$ ), particles migrate towards the channel centerline, and above a certain Re threshold, the large $(10-\mu \mathrm{m})$ particles focusing streams merge as a single beam along channel centerline. In contrast, small particles $(3-\mu \mathrm{m})$ still occupy the two sidewalls for a wider Re region, Fig. 1(c). Even above a threshold, no central focusing is observed but the defocusing emerges. Defocusing can be explained by the stirring effects of secondary flow on the small particles [38]. By employing the overlap of the central focusing status of large $(10-\mu \mathrm{m})$ particles and the two-sided focusing status of small particles $(3-\mu \mathrm{m})$, continuous and efficient separation of binary particles in the serpentine microchannel can be achieved, Fig. 1(b and d), which is the principle of WBCs separation in this work.

\section{B. Inertial Separation of Polystyrene Beads}

In whole blood, the concentration ratio of WBCs to RBCs can be as low as 1:1000, thus making efficient separation and purification of WBCs from whole blood a technical challenge. In order to characterize the separation performance of the symmetric serpentine channel, we prepared polystyrene beads mixture $(3-\mu \mathrm{m}$ and $10-\mu \mathrm{m})$ with a similar concentration ratio and infused the particle mixtures into the microchannel at the flow rate of $600 \mu 1 / \mathrm{min}(\operatorname{Re}=120)$. The concentrations of $3-\mu \mathrm{m}$ and $10-\mu \mathrm{m}$ particles are $4 \times 10^{7}$ counts $/ \mathrm{ml}$ and $4 \times 10^{4}$ counts $/ \mathrm{ml}$, respectively. After the first process through the serpentine channel, the concentration of $10-\mu \mathrm{m}$ particles from the central collection increased to $1.4 \times 10^{5}$ counts $/ \mathrm{ml}$, whereas the concentration of $3-\mu \mathrm{m}$ particles decreased to $1.19 \times 10^{6}$ counts/ml, Fig. 2(b). The optical images in Fig. 2(a) clearly show significant depletion of $3-\mu \mathrm{m}$ particles and enrichment of $10-\mu \mathrm{m}$ particles. Therefore, the purity of $10-\mu \mathrm{m}$ particles is enhanced from $0.1 \%$ to $10.59 \%$ after a process, resulting in an enrichment ratio of 100 times, Fig. 2(c).

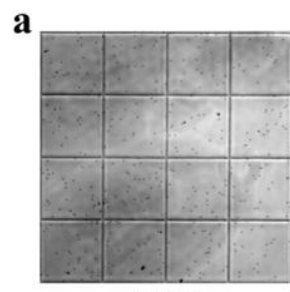

(i) Inlet

b

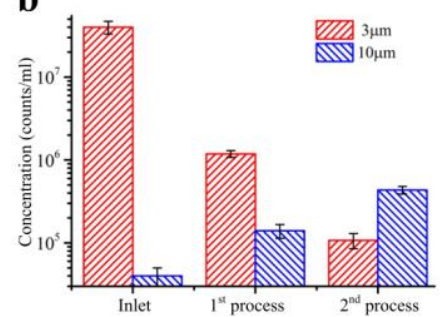

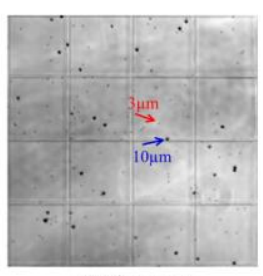

(ii) $1^{\text {st }}$ process

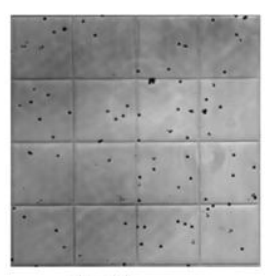

(ii) $2^{\text {nd }}$ process

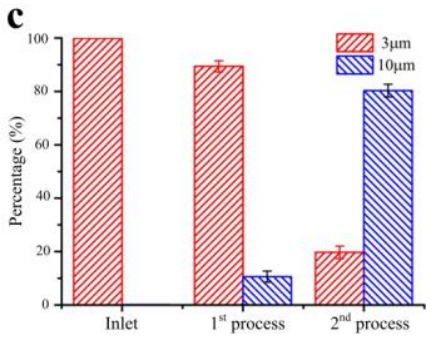

Fig. 2. Separation performance of the proposed microchannel for $3-\mu \mathrm{m}$ and 10- $\mu \mathrm{m}$ polystyrene beads. (a) Brightfield images of particle mixture at (i) inlet, and (ii) central outlet collection from the first process and (iii) central outlet collection from the second process. The input sample for the second process is the central outlet collection from the first process. (b) The concentration of particle mixture before and after each process. (c) The percentage of 3- $\mu \mathrm{m}$ and $10-\mu \mathrm{m}$ polystyrene beads before and after each process. The portion of $10-\mu \mathrm{m}$ polystyrene beads increased significantly from $0.102 \%$ to $80.31 \%$ after two cascaded processes.

After a second process using the central collection from the first one, the concentration of $10-\mu \mathrm{m}$ particles is further raised to $4.35 \times 10^{5}$ counts $/ \mathrm{ml}$, with the purity improved to $80.3 \%$, Fig. 2(b, c). Whereas, the concentration of small particles is decreased to $1.075 \times 10^{5}$ counts $/ \mathrm{ml}$, occupying only $19.7 \%$ of the total number of particles at the central outlet. In summary, the purity of $10-\mu \mathrm{m}$ particles is increased from $0.1 \%$ to $80.3 \%$ after two cascaded processes, with an average purity enrichment ratio of about 28 in each circle. Meanwhile, the separation efficiency (or recovery ratio, defined as the number ratio of collected target particles to the input target particles) of $10-\mu \mathrm{m}$ particles is more than $95 \%$ in each process.

\section{Separation of Jurkat Cells Spiked In Blood Samples}

Jurkat cells, which are an immortalized line of human $\mathrm{T}$ lymphocytes, were used to investigate the inertial focusing behavior of cells in the symmetric serpentine microchannel. Jurkat cells were cultured and stained using CellTracker Green CMFDA to allow visualization of cell trajectory, Fig. 3(a). The diameter of Jurkat cells is $14.5 \pm 2.2 \mu \mathrm{m}(\mathrm{n}=167)$. Jurkat cell suspensions were infused into the symmetric serpentine 
channel at flow rates from $200 \mu \mathrm{l} / \mathrm{min}$ to $900 \mu \mathrm{l} / \mathrm{min}$. Their focusing positions at the end of the serpentine channel were captured, Fig. 3b. We can see that the focusing phenomenon of Jurkat cells is similar to that of the rigid polystyrene beads observed above: the two-sided focusing streaks migrated toward the channel centerline with an increment of flow rate and finally merged as a single streak at the centerline. This can be further validated by the fluorescent profile, Fig. 3(c).

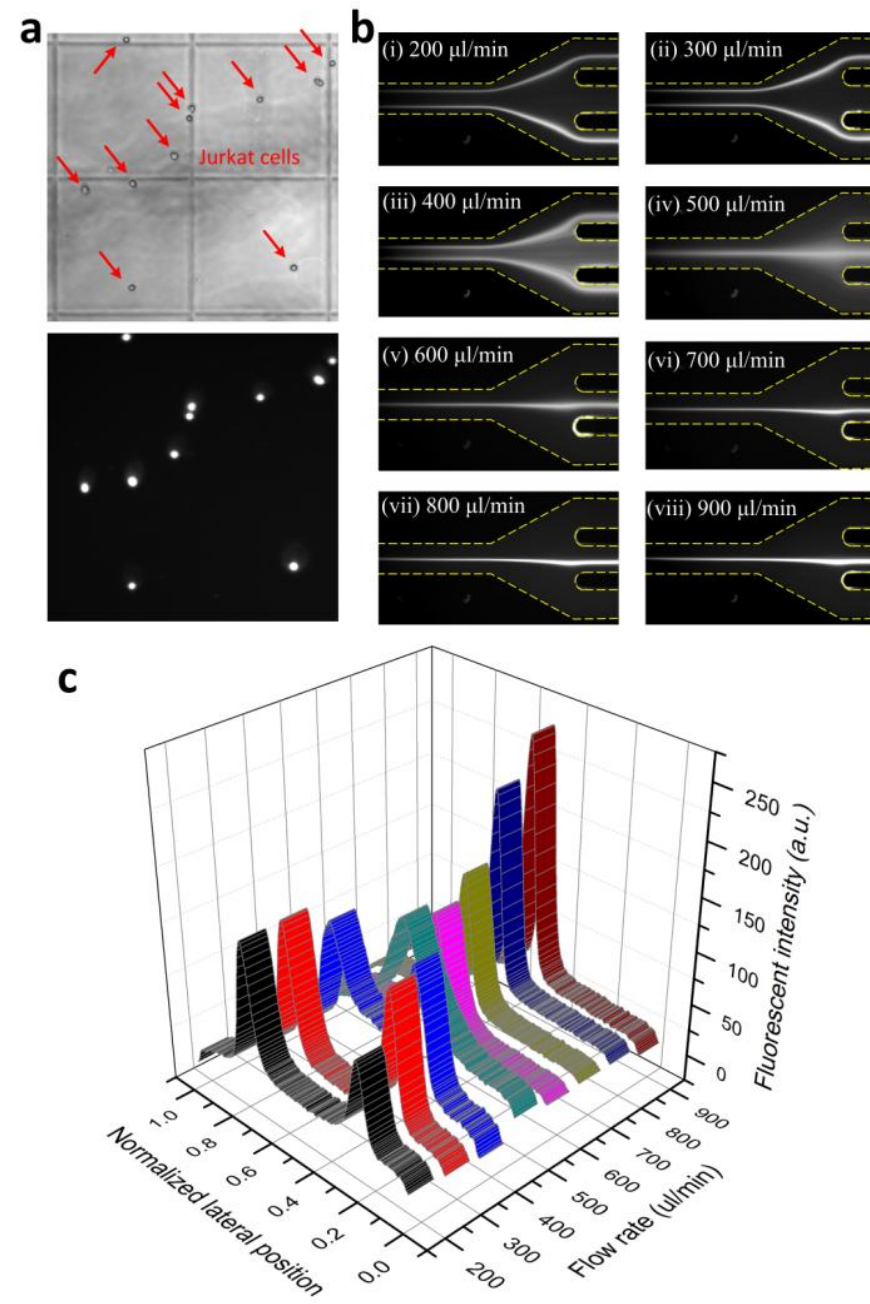

Fig. 3. Inertial focusing of Jurkat cells in the symmetrical serpentine microchannel. (a) Brightfield (top) and fluorescent (bottom) images of the stained Jurkat cells. (b) Inertial focusing positions of Jurkat cells at flow rates ranging from $200 \mu \mathrm{1} / \mathrm{min}$ to $900 \mu \mathrm{1} / \mathrm{min}$. (c) Fluorescent profiles of cell focusing stream at the end of the serpentine microchannel.

To mimic the whole blood and further evaluate the inertial focusing and separation of Jurkat cells in the presence of blood cells, the Jurkat cells were spiked into the blood in a number ratio of about $0.1 \%$ to mimic the behavior of WBCs in the whole blood. The brightfield and fluorescent images, which were for the better observation of RBCs and the stained Jurkat cells respectively, were composited to verify their distinguished focusing positions, Fig. 4(a). The RBCs (the dark streaks) are obviously focused along two sidewalls, whereas the Jurkat cells (the white streak) are concentrated tightly along the channel centerline. We also investigated the effects of flow rates on their focusing status, Fig. 4(b). The optimal flow rate was around $600 \mu \mathrm{l} / \mathrm{min}$, when the flow rate was too low (400 $\mu \mathrm{l} / \mathrm{min}$ ), Jurkat cells could not focus tightly along the centerline, and some exited from the up and bottom outlets. However, if the flow rate was too high $(800 \mu 1 / \mathrm{min})$, the RBCs defocused and occupied a wide region of channel width due to the mixing effects of the secondary flow. In this case, large amount of RBCs will follow Jurkat cells, entering the central outlet.

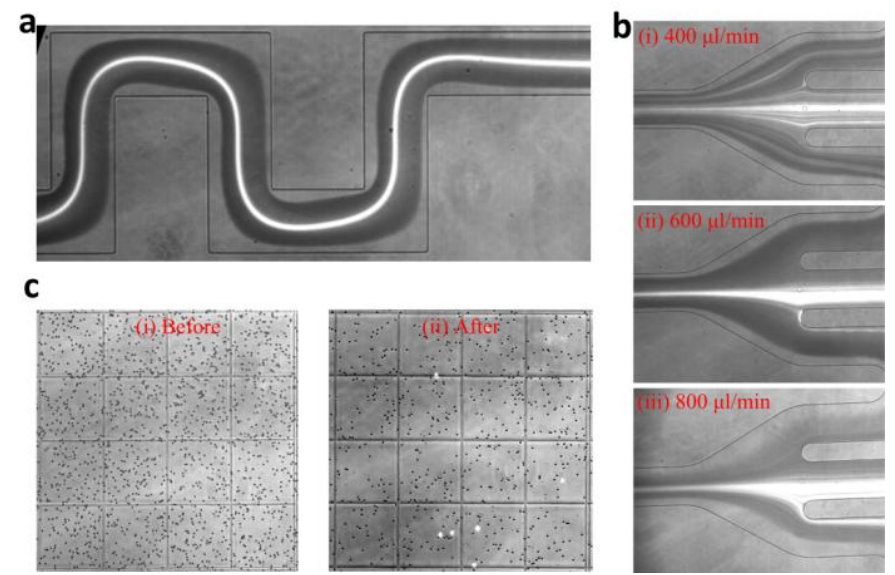

Fig. 4. (a) Inertial focusing and separation of Jurkat cells spiked in the diluted $(\times 1 / 20)$ whole blood. (b) Inertial focusing positions and distribution of stained Jurkat cells and blood cells at flow rates of (i) $400 \mu 1 / \mathrm{min}$, (ii) $600 \mu \mathrm{l} / \mathrm{min}$, and (iii) $800 \mu \mathrm{l} / \mathrm{min}$. (c) The optical images of Jurkat cells spiked in the blood under a hemocytometer before and after the process by the inertial microfluidic device. The dark points are blood cells, and the bright points are the stained Jurkat cells. The ratio of spiked Jurkat cells is increased by 11 times, from $0.1 \%$ to $1.1 \%$.

At an optimal flow rate, the RBCs can be depleted and Jurkat cells can be concentrated significantly at the central outlet, Fig. 4(c). The ratio of spiked Jurkat cells in the blood is enhanced from $0.1 \%$ to $1.1 \%$ after a single process, with an enrichment ratio of 11 times. It is noted that this value is significantly lower than that of polystyrene beads, for which the enrichment ratio for the average enrichment ratio is 28 times. This is due to the fact that intensive cell-cell interactions in the blood sample deteriorate the cells focusing and separation performance. Although the blood sample was diluted $\times 1 / 20$ using PBS, the concentration of cells was still in the order of $10^{8}$ counts $/ \mathrm{ml}$, which was one order of magnitude higher than that of polystyrene beads sample.

\section{Separation of WBCs From The Whole Blood}

From the above characterization of separation performance using polystyrene beads mixture and Jurkat cells spiked in blood, we found that the purity of target cells is unsatisfactory after single process, so a cascaded procedure that further processes the targeted central collection from the previous one is needed, Fig. 5(a). Meanwhile, the dilution of the whole blood sample is required, which will reduce the net flow rate, therefore a parallelization design to amplify the whole throughput is desired. Here, we designed and fabricated a parallelized device with eight parallel serpentine channels, Fig. 5(b). The flow rate of diluted $(\times 1 / 20)$ whole blood infused into the device is $288 \mathrm{ml} / \mathrm{h}$, equivalent to $14.4 \mathrm{ml} / \mathrm{h}$ for the undiluted blood. The throughput can be further enlarged by stacking several layers of microchannels along the height direction.

The input blood sample and central collections from two consecutive processes were tested by a flow cytometry, Fig. 5(c). The separation purity of WBCs increased to about $48 \%$ 
after two processes, with an average enrichment ratio of about 10 times, which is sufficient for many clinical assays [8]. We observed that the separation purity of WBCs after each process was lower than that of polystyrene beads separation. In addition to the intensive cell-cell interaction as discussed above, a wide size distribution of WBCs, ranging from $\sim 8 \mu \mathrm{m}$ in diameter (small $\mathrm{T}$ and B lymphocytes) to $\sim 11 \mu \mathrm{m}$ (small granulocytes like neutrophils and eosinophils) and $\sim 14 \mu \mathrm{m}$ and above for the largest subpopulations (large T and B lymphocytes, monocytes, and larger granulocytes like basophils) [14], may also contribute to the degradation of separation performance.
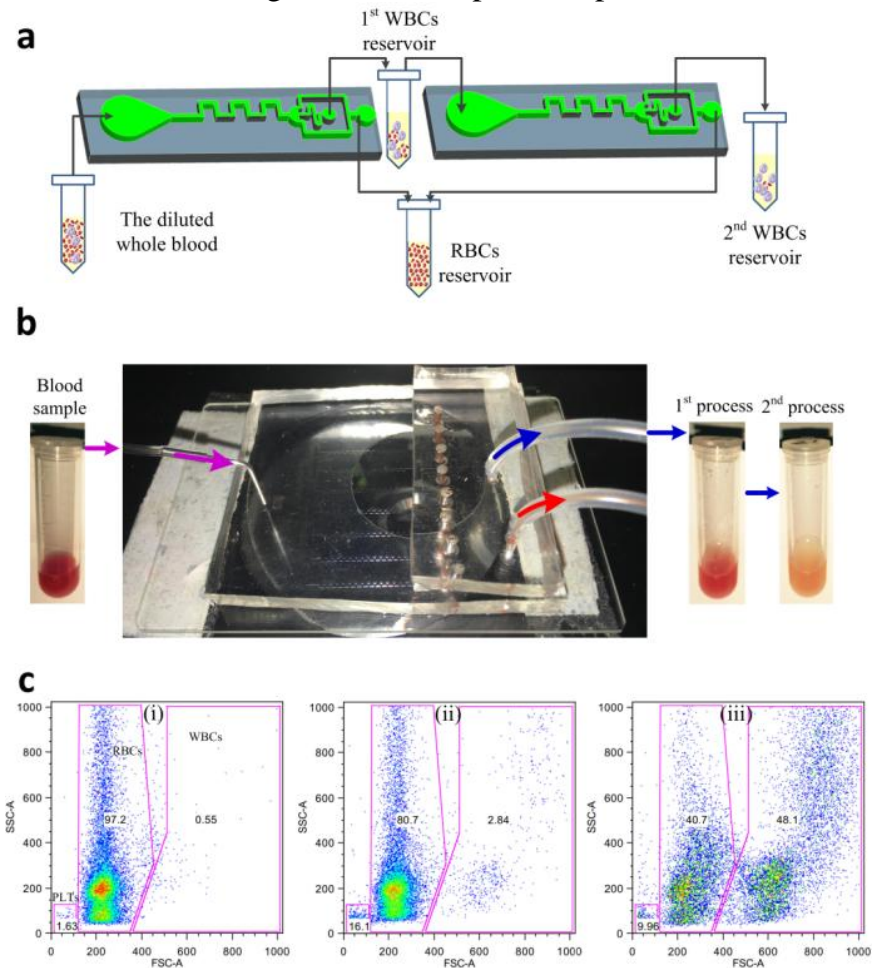

Fig. 5. (a) A schematic of cascaded separation and purification of white blood cells (WBCs) from whole blood. (b) A picture of the working inertial microfluidic device with eight parallel serpentine microchannels and optical images of the input blood sample and collections in the tubes after two cascaded processes. (c) Flow cytometry data indicate the percentage of white blood cells (WBCs), red blood cells (RBCs) and platelets (PLTs) numbers in the (i) whole blood sample, (ii) central collection after the first process, and (iii) central collection after the second process.

The proposed microfluidic device holds several advantages. First, there is no clogging issue in the proposed method compared to the filtration based methods [17, 19]. Second, compared with the reported inertial microfluidic systems, which process highly diluted blood sample $(\times 1 / 400-\times 1 / 200)$ [27, 28], the proposed microfluidic device can handle much less $(\times 1 / 20)$ diluted blood sample. Third, the proposed methods can provide a high throughput. The equivalent throughput of the proposed device for the undiluted blood is $240 \mu \mathrm{l} / \mathrm{min}$, which is 30 times that of DEP microseparators [12], 48 times that of paramagnetic capture (PMC) mode microseparators [15], 14-1000 times that of cross flow filtrations [17-19], 1.6-800 times that of margination-based techniques $[8,21]$, and 9-66 times that of the-state-of-art inertial micofluidic devices [27-29].

Table 1 summarizes the WBCs separation performance of different microfluidic methods. It should be noted that the shear stress may affect the functionality and viability of WBCs in the separation process. In this work, WBCs are focusing at the center of microchannel where shear rate is minimum, with an order same as that in Kim's work [21], where WBCs are focused along one sidewall of the microchannel with the shear rate at $1.3 \times 10^{4} \mathrm{~s}^{-1}$. In their work, the shear rate did not impair the integrity and function of WBCs. Besides, the duration of WBCs in the microfluidic device is very short, only about 15 $\mathrm{ms}$. Therefore, the effect of shear rate on the functionality and viability on WBCs is believed negligible. This can be further supported by our previous work using the same device for separation of neuron and glial cells [41].

Table 1 Comparison of different microfluidic methods on WBCs separation.

\begin{tabular}{|c|c|c|c|c|c|}
\hline Methods & Sample & Flow rate & Purity & $\begin{array}{c}\text { Efficie } \\
\text { ncy }\end{array}$ & Refs \\
\hline $\begin{array}{c}\text { Dielectrop } \\
\text { horesis }\end{array}$ & (1/6) diluted & $50 \mu 1 / \min$ & N/A & $\begin{array}{l}92.1 \%, \\
76.9 \% \\
\end{array}$ & [12] \\
\hline $\begin{array}{l}\text { Paramagne } \\
\text { tic capture }\end{array}$ & Undiluted & $5 \mu \mathrm{l} / \mathrm{h}$ & N/A & $97.4 \%$ & [15] \\
\hline \multirow{3}{*}{$\begin{array}{l}\text { Cross flow } \\
\text { filtration }\end{array}$} & (1/10) diluted & $20 \mu 1 / \min$ & $3.71 \%$ & N/A & [17] \\
\hline & Undiluted & $1 \mathrm{ml} / \mathrm{h}$ & $93.5 \%$ & $27.4 \%$ & [19] \\
\hline & (1/20) diluted & $40 \mu 1 / \min$ & $\sim 95 \%$ & NA & [20] \\
\hline \multirow{2}{*}{$\begin{array}{c}\mathrm{WBC} \\
\text { marginatio } \\
\mathrm{n}\end{array}$} & Undiluted & $18 \mu \mathrm{l} / \mathrm{h}$ & $5 \%$ & $94 \%$ & {$[8]$} \\
\hline & Undiluted & $150 \mu 1 / \mathrm{min}$ & $\sim 10 \%$ & $80 \%$ & {$[21]$} \\
\hline \multirow{4}{*}{$\begin{array}{l}\text { Inertial } \\
\text { microfluidi } \\
\text { cs }\end{array}$} & $\begin{array}{l}(1 / 400) \\
\text { diluted }\end{array}$ & $10.8 \mathrm{ml} / \mathrm{min}$ & $91 \%$ & $89.7 \%$ & [27] \\
\hline & $\begin{array}{l}(1 / 500) \\
\text { diluted }\end{array}$ & $1.8 \mathrm{ml} / \mathrm{min}$ & N/A & $95 \%$ & {$[28]$} \\
\hline & (1/9) diluted & $900 \mu \mathrm{l} / \mathrm{min}$ & N/A & $98.4 \%$ & [29] \\
\hline & (1/20) diluted & $288 \mathrm{ml} / \mathrm{h}$ & $48.1 \%$ & $>90 \%$ & $\begin{array}{l}\text { This } \\
\text { work }\end{array}$ \\
\hline
\end{tabular}

In this work, we manually collected the central collection after the first process and infused into the same device to conduct the second round. It could also be carried out by connecting the central outlet of the first device with the input of the second one in a cascaded way to avoid human intervention. In this case, a sheath flow or a delicate outlet design is required to adjust the flow rate and stream division in each serpentine microchannel, ensuring every channel working at the optimal status.

\section{CONCLUSIONS}

In this work, we developed a continuous and high-throughput microfluidic platform for WBCs separation utilizing the symmetric serpentine microchannel, which provides differential inertial focusing of WBCs and RBCs along the lateral direction. Inertial focusing and separation of polystyrene beads and mammalian cells in the serpentine channel were conducted to characterize separation performance of the channel. The purity of $10-\mu \mathrm{m}$ polystyrene beads was increased from $0.1 \%$ to $80.3 \%$ from after two processes, with the average purity enrichment ratio of 28 times. The purity of WBCs from the whole blood was increased to $48 \%$ after two consecutive processes, with an average enrichment ratio of 10 times. Also, we designed and fabricated a parallelized inertial microfluidic device, which can provide a high processing flow rate of $288 \mathrm{ml} / \mathrm{h}$ for the diluted $(\times 1 / 20)$ whole blood, equivalent to $14.4 \mathrm{ml} / \mathrm{h}$ for the undiluted whole blood. The proposed 
inertial microfluidic device can be applied to the high volume blood sample preparation and analysis in the upstream of an integrated microfluidic system.

\section{REFERENCES}

[1] J. Guo, "Uric Acid Monitoring with Smartphone as Electrochemical Analyzer," Analytical Chemistry, vol. 88, pp. 11986-11989, 2016.

[2] J. Guo and X. Ma, "Simultaneous monitoring of glucose and uric acid on a single test strip with dual channels," Biosensors \& Bioelectronics, vol. 94, pp. 415-419, 2017.

[3] R. S. Vasan, "Biomarkers of cardiovascular disease: molecular basis and practical considerations," Circulation, vol. 113, pp. 2335-2362, 2006.

[4] W. Chen, "Emerging Microfluidic Tools for Functional Cellular Immunophenotyping: A New Potential Paradigm for Immune Status Characterization," Frontiers in Oncology, vol. 3, Art No. 98, 2013.

[5] Q. Zhou, T. Kwa, Y. Liu, and A. Revzin, "Cytokine biosensors: the future of infectious disease diagnosis?," Expert Review of Anticancer Therapy, vol. 10, pp. 1079-1081, 2012.

[6] A. A. S. Bhagat, H. W. Hou, L. D. Li, C. T. Lim, and J. Han, "Pinched flow coupled shear-modulated inertial microfluidics for high-throughput rare blood cell separation," Lab on a Chip, vol. 11, pp. 1870-1878, 2011.

[7] D. Di Carlo, F. Jon, D. Irimia, R. G. Tompkins, and M. Toner, "Equilibrium separation and filtration of particles using differential inertial focusing," Analytical Chemistry, vol. 80, pp. 2204-2211, 2008.

[8] A. Jain and L. L. Munn, "Biomimetic postcapillary expansions for enhancing rare blood cell separation on a microfluidic chip," Lab on A Chip, vol. 11, pp. 2941-2947, 2011.

[9] S. Fukuda and G. W. Schmid-Schönbein, "Centrifugation attenuates the fluid shear response of circulating leukocytes," Journal of leukocyte biology, vol. 72, pp. 133-139, 2002.

[10] Y. Xie, D. Zheng, Q. Li, Y. Chen, H. Lei, and L. L. Pu, "The effect of centrifugation on viability of fat grafts: an evaluation with the glucose transport test," Journal of Plastic, Reconstructive \& Aesthetic Surgery, vol. 63, pp. 482-487, 2010.

[11] K. S. Lang, P. A. Lang, C. Bauer, C. Duranton, T. Wieder, S. M. Huber, et al., "Mechanisms of suicidal erythrocyte death," Cellular Physiology \& Biochemistry International Journal of Experimental Cellular Physiology Biochemistry \& Pharmacology, vol. 15, pp. 195-202, 2005.

[12] K. H. Han and A. B. Frazier, "Lateral-driven continuous dielectrophoretic microseparators for blood cells suspended in a highly conductive medium," Lab on A Chip, vol. 8, pp. 1079-1086, 2008.

[13] Filip Petersson, Lena Åberg, A. Annmargret Swärdnilsson, and Thomas Laurell, "Free flow acoustophoresis: microfluidic-based mode of particle and cell separation," Analytical Chemistry, vol. 79, pp. 5117-5123, 2007.

[14] C. Grenvall, C. Magnusson, H. Lilja, and T. Laurell, "Concurrent isolation of lymphocytes and granulocytes using prefocused free flow acoustophoresis," Analytical chemistry, vol. 87, pp. 5596-5604, 2015.

[15] K. H. Han and A. B. Frazier, "Paramagnetic capture mode magnetophoretic microseparator for high efficiency blood cell separations," Lab on A Chip, vol. 6, pp. 265-273, 2006.

[16] C. Iliescu, E. Barbarini, M. Avram, and G. Xu, "Microfluidic device for continuous magnetophoretic separation of red blood cells," Microsystem Technologies, vol. 15, pp. 1157-1162, 2009.

M. Yamada and M. Seki, "Hydrodynamic filtration for on-chip particle concentration and classification utilizing microfluidics," Lab on A Chip, vol. 5, pp. 1233-1239, 2005.

[18] V. VanDelinder and A. Groisman, "Perfusion in microfluidic cross-flow: separation of white blood cells from whole blood and exchange of medium in a continuous flow," Analytical Chemistry, vol. 79, pp. 2023-2030, 2007.

[19] X. Li, W. Chen, G. Liu, W. Lu, and J. Fu, "Continuous-flow microfluidic blood cell sorting for unprocessed whole blood using surface-micromachined microfiltration membranes," Lab on a Chip, vol. 14, pp. 2565-2575, 2014.

[20] M. Yamada, W. Seko, T. Yanai, K. Ninomiya, and M. Seki, "Slanted, asymmetric microfluidic lattices as size-selective sieves for continuous particle/cell sorting," Lab on a Chip, vol. 17, pp. 304-314, 2017.

B. Kim, Y. J. Choi, H. Seo, E. C. Shin, and S. Choi, "Deterministic Migration-Based Separation of White Blood Cells," Small, vol. 12, pp. 5159-5168, 2016.

[22] D. Di Carlo, "Inertial microfluidics," Lab on a Chip, vol. 9, pp. 3038-3046, 2009.

[23] J. Zhang, S. Yan, D. Yuan, G. Alici, N.-T. Nguyen, M. Ebrahimi Warkiani, et al., "Fundamentals and applications of inertial microfluidics: a review," $L a b$ on a Chip, vol. 16, pp. 10-34, 2016.

[24] Q. Chen, D. Li, J. Lin, M.-H. Wang, and X. Xuan, "Simultaneous separation and washing of non-magnetic particles in an inertial ferrofluid/water co-flow," Analytical Chemistry, vol. 89, pp. 6915-6920, 2017.

[25] Y. Zhou, L. Song, L. Yu, and X. Xuan, "Inertially focused diamagnetic particle separation in ferrofluids," Microfluidics and Nanofluidics, vol. 21, Art No. 14, 2017.

[26] W. Tang, D. Tang, Z. Ni, N. Xiang, and H. Yi, "Microfluidic Impedance Cytometer with Inertial Focusing and Liquid Electrodes for High-Throughput Cell Counting and Discrimination," Analytical Chemistry, vol. 89, pp. 3154-3161, 2017.

[27] Z. Wu, Y. Chen, M. Wang, and A. J. Chung, "Continuous inertial microparticle and blood cell separation in straight channels with local 
microstructures," Lab on a Chip, vol. 16, pp. 532-542, 2016.

[28] N. Nivedita and I. Papautsky, "Continuous separation of blood cells in spiral microfluidic devices," Biomicrofluidics, vol. 7, Art No. 054101, 2013.

[29] L. Wu, G. Guan, H. W. Hou, A. A. S. Bhagat, and J. Han, "Separation of Leukocytes from Blood Using Spiral Channel with Trapezoid Cross-Section," Analytical Chemistry, vol. 84, pp. 9324-9331, 2012.

[30] D. C. Duffy, J. C. McDonald, O. J. A. Schueller, and G. M. Whitesides, "Rapid prototyping of microfluidic systems in poly (dimethylsiloxane)," Analytical Chemistry, vol. 70, pp. 4974-4984, 1998.

[31] E. S. ASMOLOV, "The inertial lift on a spherical particle in a plane Poiseuille flow at large channel Reynolds number," Journal of Fluid Mechanics, vol. 381, pp. 63-87, 1999.

[32] A. A. S. Bhagat, S. S. Kuntaegowdanahalli, N. Kaval, C. J. Seliskar, and I. Papautsky, "Inertial microfluidics for sheath-less high-throughput flow cytometry," Biomedical microdevices, vol. 12, pp. 187-195, 2010.

[33] E. W. Kemna, R. M. Schoeman, F. Wolbers, I. Vermes, D. A. Weitz, and A. van den Berg, "High-yield cell ordering and deterministic cell-in-droplet encapsulation using Dean flow in a curved microchannel," Lab on a Chip, vol. 12, pp. 2881-2887, 2012.

[34] S. Berger, L. Talbot, and L. Yao, "Flow in curved pipes," Annual review of fluid mechanics, vol. 15, pp. 461-512, 1983.

[35] S. S. Kuntaegowdanahalli, A. A. S. Bhagat, G. Kumar, and I. Papautsky, "Inertial microfluidics for continuous particle separation in spiral microchannels," Lab Chip, vol. 9, pp. 2973-2980, 2009.

[36] D. Di Carlo, D. Irimia, R. G. Tompkins, and M. Toner, "Continuous inertial focusing, ordering, and separation of particles in microchannels," Proceedings of the National Academy of Sciences, vol. 104, pp. 18892-18897, 2007.

[37] J. Zhang, S. Yan, W. Li, G. Alici, and N.-T. Nguyen, "High throughput extraction of plasma using a secondary flow-aided inertial microfluidic device," RSC Advances, vol. 4, pp. 33149-33159, 2014.

[38] J. Zhang, S. Yan, R. Sluyter, W. Li, G. Alici, and N.-T. Nguyen, "Inertial particle separation by differential equilibrium positions in a symmetrical serpentine micro-channel," Scientific reports, vol. 4, Art No. 4527, 2014.

[39] V. VanDelinder and A. Groisman, "Separation of plasma from whole human blood in a continuous cross-flow in a molded microfluidic device," Analytical chemistry, vol. 78, pp. 3765-3771, 2006.

[40] M. Toner and D. Irimia, "Blood-on-a-chip," Annual review of biomedical engineering, vol. 7, pp. 77-103, 2005.

[41] T. Jin, S. Yan, J. Zhang, D. Yuan, X.-F. Huang, and W. $\mathrm{Li}$, "A label-free and high-throughput separation of neuron and glial cells using an inertial microfluidic platform," Biomicrofluidics, vol. 10, Art No. 034104, 2016.

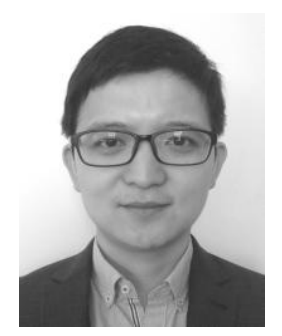

Jun Zhang received his bachelor degree in Engineering from the Nanjing University of Science and Technology (NJUST), Nanjing, China in 2009 with an Outstanding Graduate Award and received a Ph.D. degree in Mechanical Engineering from the University of Wollongong, Australia in 2015.

After that, he conducted his postdoctoral research as a research scientist and an associate research fellow at Woolcock Institute of Medical Research and the University of Wollongong, respectively. He is currently an Associate Professor at Nanjing University of Science and Technology (NJUST), China. He has published more than 30 journal and conference articles.

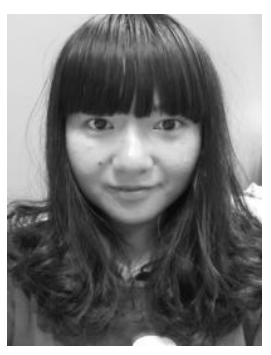

Dan Yuan was born in Shandong province, China, in 1990. She received her Bachelor degree in Engineering and Master degrees in Engineering from Hefei University of Technology in Hefei, China, in 2011 and 2014, and majored in Precision Measurement Technology and Instruments.

Since 2014, she has been a PhD candidate in the School of Mechanical, Materials, Mechatronic and Biomedical Engineering at University of Wollongong. She is the author of more than 18 papers. Her research interests are particle and cell manipulation in viscoelastic fluid.

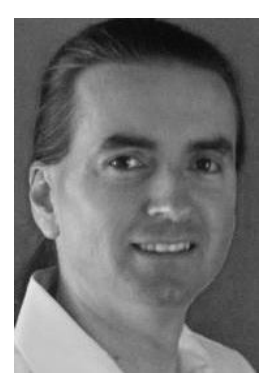

Ronald Sluyter received the B.App.Sc. degree in biomedical science from the University of Technology, Sydney, Australia in 1991, and the Ph.D. degree in medicine from the University of Sydney, Sydney, Australia in 1999.

From 1992 to 1994, he was a Research Assistant with the University of Sydney. From 2000 to 2007, he was a Research Officer with the Nepean Hospital, Penrith, Australia. From 2008 to 2014 he was a Senior Lecturer with the University of Wollongong, Wollongong, Australia. Since 2015 he has been an Associate Professor with the University of Wollongong. He has coauthored more than 76 articles. His research interests include purinergic signaling, inflammation and immunity, cell biology, and cell separation techniques.

Assoc. Prof. Sluyter is a current member of the Australian Society for Immunology and the Australian Physiology Society.

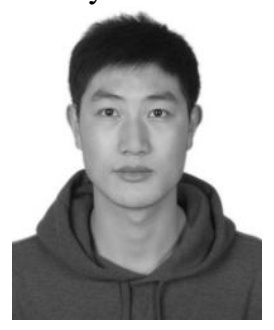

Sheng Yan received his Bachelor degree in Engineering with an Outstanding Graduate Award from 
China University of Mining and Technology in 2012 and a doctoral degree from the University of Wollongong in 2016. He currently works as an associate research fellow in the School of Mechanical, Materials and Mechatronic Engineering at the University of Wollongong. His main research focus is to develop hybrid techniques to tune hydrophoresis for cell focusing and separation. His research interests include dielectrophoresis, hydrophoresis, magnetophoresis, neurosciences, computational fluid dynamics (CFD).

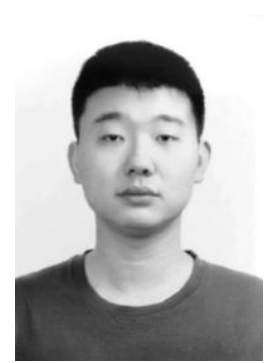

Qianbin Zhao received his bachelor's degree in engineering from the Northeastern University (NEU), Shenyang, China, in 2015. He recently holds a position as a Ph.D. candidate in the School of Mechanical, Materials, Mechatronic and Biomedical Engineering at the University of Wollongong. His current research focuses on (i) inertial microfluidics for microparticle manipulation through geometry-induced secondary flow and (ii) viscoelastic microfluidics applications for bioassays and biosample processing.

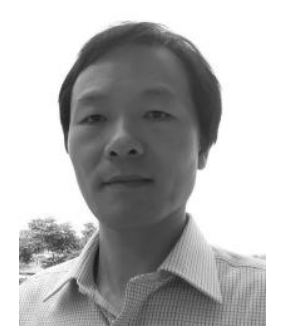

Huanming Xia is currently in the School of Mechanical Engineering, Nanjing University of Science and Technology. He received his B.Eng. and M.Eng. degrees in thermal engineering from Nanjing University of Aeronautics \& Astronautics, China, 1998, 2001, and a Ph.D. degree from National University of Singapore, Singapore, 2010. From 2007 to 2014, he worked in Singapore Institute of Manufacturing Technology (SIMTech) as a Research Scientist. His research interests include fluid dynamics, heat and mass transfer, microfluidics, microreactor technology and micro-fabrication techniques.

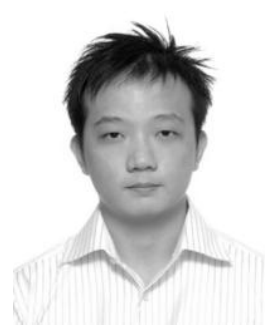

Say Hwa Tan is an ARC DECRA fellow with Queensland Micro-and Nanotechnology Centre, Griffith University, Australia. He received his $\mathrm{BEng}$, MEng and $\mathrm{PhD}$ degrees from the Nanyang Technological University, Singapore, and the Georg-August-Universität Göttingen/Max Planck Institute for dynamics and self-organization (MPI-DS), Germany, in 2008, 2010 and 2014 respectively. In 2016, he was highlighted as one of the 18 emerging investigators in the journal of Lab on a Chip. Dr Tan has published more than 30 research works in microfluidics.
His research has established and pioneered different approaches to manipulate droplets and bubbles using thermal, magnetic, acoustic, pneumatic and electric energy.

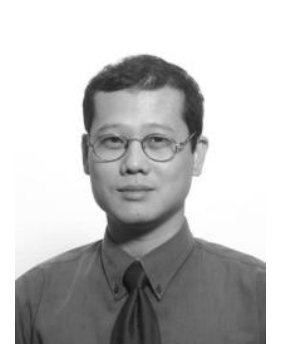

Nam-Trung Nguyen received his Dip-Ing, Dr. Ing and Dr. Ing Habil degrees from Chemnitz University of Technology, Germany, in 1993, 1997 and 2004, respectively. The habilitation degree (Dr. Ing Habil) is the qualification for a full professorship in Germany. In 1998, he was a postdoctoral research engineer in the Berkeley Sensor and Actuator Center (University of California at Berkeley, USA). Prof Nguyen is the First Runner Up of Inaugural ProSPER.Net-Scopus Young Scientist Awards in Sustainable Development in 2009 and the Runner Up of ASAIHL-Scopus Young Scientist Awards in 2008. He is a Fellow of ASME and a Member of IEEE. Nguyen's research is focused on microfluidics, nanofluidics, micro/nanomachining technologies, micro/nanoscale science, and instrumentation for biomedical applications. He published over 300 journal papers and filed 8 patents, of which 3 were granted. Among the books he has written, the first and second editions of the bestseller "Fundamentals and Applications of Microfluidics" were published in 2002 and 2006, respectively. The second edition of the bestselling book "Micromixer" was acquired and published by Elsevier in 2011.

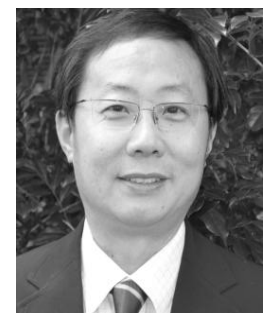

Weihua Li, Ph.D., is a Senior Professor and Director of the Advanced Manufacturing Research Strength at the University of Wollongong. He completed his BEng (1992), MEng (1995) at University of Science and Technology of China, and Ph.D. (2001) at Nanyang Technological University (NTU). He was with the School of Mechanical and Aerospace Engineering of NTU as a Research Associate/Fellow from 2000 to 2003, before he joined the School of Mechanical, Materials and Mechatronic Engineering as a Lecturer. His research focuses on smart materials and their applications, microfluidics, rheology, and intelligent mechatronics. He is serving as editor or editorial board member for several international journals, including IEEE/ASME Transactions on Mechatronics, Smart Materials and Structures, Scientific Reports, etc. He has published more than 320 journal and conference papers. He is a recipient of Fellow of the Engineers Australia, the Fellow of Institute of Physics (UK), Australian Endeavour Research Fellowship, JSPS Invitation Fellowship, Vice-chancellor's Award for Interdisciplinary Research Excellence, and numerous Best Paper Awards. 